\title{
A Novel Multifunction CFOA-Based Inverse Filter
}

\author{
Hung-Yu Wang ${ }^{1}$, Sheng-Hsiung Chang ${ }^{2}$, Tzu-Yi Yang ${ }^{1}$, Po-Yang Tsai ${ }^{1}$ \\ ${ }^{1}$ Department of Electronic Engineering, National Kaohsiung University of \\ Applied Sciences, Kaohsiung, Taiwan, China \\ ${ }^{2}$ Department of Optoelectronic Engineering, Far East University, Hsin-Shih, Taiwan, China \\ E-mail: hywang@cc.kuas.edu.tw \\ Received October 7, 2010; revised November 3, 2010; accepted November 17, 2010
}

\begin{abstract}
We present a novel multifunction inverse biquad configuration based on current feedback operational amplifiers (CFOAs) and grounded passive elements. The proposed scheme can be used to realize inverse lowpass, inverse bandpass and inverse highpass filter functions. The relevant coefficients of the inverse filters are orthogonal adjustable by independent passive elements. All the passive elements in the proposed scheme are grounded to benefit easier electronic tunability. With the high input impedance and low output impedance properties, the scheme is input and output cascadable for voltage operation. The feasibility of the proposed scheme is demonstrated by HSPICE simulations.
\end{abstract}

Keywords: Multifunction, Inverse Filter, CFOA

\section{Introduction}

In communication, control and instrumentation systems, there are numerous situations in which an electrical signal is altered through a linear or nonlinear transformation by a processing or a transmission system. So it is necessary to recover the input signal from the available distorted output signal resulted from the signal progress. This can often be done by using a system that has an inverse transfer characteristic of the original system [1]. For digital signal processing, several methods for obtaining digital inverse filters have been established [2]. Nevertheless, for analog signal processing, only a few works are known for realizing continuous-time analog inverse filters [1,3-6].

In [1], a general approach is presented for obtaining the inverse transfer function for linear dynamic systems and the inverse transfer characteristic for non-linear resistive circuits. In [3], a procedure for deriving current-mode, four-terminal floating nullor (FTFN)-based inverse filter from the voltage-mode filter is given. It uses the method in [1] and dual transformation [7] during the procedure. Due to the use of dual transformation, this approach can only be applied to planar circuit. By the use of adjoint transformation, another easier procedure for deriving current-mode FTFN-based inverse filter from the voltage-mode filter is presented and it is applicable to nonplanar circuits [4]. All the proposed approaches in
$[1,3,4]$ are useful for obtaining single-input single-output inverse filters. Additional various inverse current-mode and voltage-mode filters are presented in [5] and [6], respectively. However, each circuit proposed in $[5,6]$ has one inverse filter function. In this paper, we present a novel inverse filter scheme based on CFOAs and grounded passive elements. By slight modification of the passive elements of the proposed scheme, various inverse filter functions can be realized. The presented scheme possesses high input impedance and low output impedance which enables the convenience of connecting with the other stage in cascade. The workability of the proposed scheme is verified by HSPICE simulations. The simulated results confirm the theoretical prediction.

\section{The Proposed Circuit}

The current-feedback operational amplifier, such as AD844 from Analog Devices Inc. [8], has gained the acceptance of researchers as a building block in circuit design. The advantages of CFOAs are their constant bandwidths, independent closed-loop gains and high slew-rate capabilities [9]. The CFOA can be described using the following matrix-relations:

$$
\left[\begin{array}{c}
V_{x} \\
I_{y} \\
I_{z} \\
V_{w}
\end{array}\right]=\left[\begin{array}{llll}
0 & 1 & 0 & 0 \\
0 & 0 & 0 & 0 \\
1 & 0 & 0 & 0 \\
0 & 0 & 1 & 0
\end{array}\right]\left[\begin{array}{c}
I_{x} \\
V_{y} \\
V_{z} \\
I_{w}
\end{array}\right] .
$$


Considering the proposed scheme in Figure 1, three CFOAs are used to construct the circuit functions. The transfer functions can be expressed as:

$$
\begin{gathered}
\frac{V_{o 1}}{V_{\text {in }}}=\frac{V_{o 3}}{V_{\text {in }}}=\frac{y_{1} y_{3}+y_{2} y_{4}}{y_{0} y_{4}} \\
\frac{V_{o 2}}{V_{\text {in }}}=\frac{y_{1}}{y_{4}}
\end{gathered}
$$

If the admittances are $y_{0}=G_{0}, y_{1}=s C_{1}, y_{2}=s C_{2}+G_{2}, y_{3}$ $=s C_{3}$ and $y_{4}=G_{4}$, the functions of inverse lowpass filter and inverse integrator can be realized at $V_{o 1}$ and $V_{o 2}$, respectively. They are given by

$$
\begin{gathered}
\frac{V_{o 1}}{V_{\text {in }}}=\frac{V_{o 3}}{V_{\text {in }}}=\frac{s^{2} C_{1} C_{3}+s C_{2} G_{4}+G_{2} G_{4}}{G_{0} G_{4}} \\
\frac{V_{o 2}}{V_{\text {in }}}=\frac{s C_{1}}{G_{4}}
\end{gathered}
$$

From Equation (4), it is clear that the coefficients of the $s^{2}, s^{1}$ and $s^{0}$ terms in the numerator and the term in denominator are tunable by the values of $C_{1}, C_{2}, G_{2}$ and $G_{0}$ respectively. So the system parameters, such as the corner angular frequency $\omega_{o}$ and quality factor $Q$ of the inverse filter are tunable by independent passive elements.

In Equation (2), if the admittances are $y_{0}=s C_{0}, y_{1}=$ $s C_{1}, y_{2}=s C_{2}+G_{2}, y_{3}=s C_{3}$ and $y_{4}=G_{4}$, the functions of inverse bandpass filter and inverse integrator can be realized at $V_{o 1}$ and $V_{o 2}$, respectively. They can be given by

$$
\begin{gathered}
\frac{V_{o 2}}{V_{i n}}=\frac{s C_{1}}{G_{4}} \\
\frac{V_{o 1}}{V_{\text {in }}}=\frac{V_{o 3}}{V_{\text {in }}}=\frac{s^{2} C_{1} C_{3}+s C_{2} G_{4}+G_{2} G_{4}}{s C_{0} G_{4}}
\end{gathered}
$$

Similarly, if the admittances are $y_{0}=s C_{0}, y_{1}=G_{1}, y_{2}=$ $s C_{2}+G_{2}, y_{3}=G_{3}$ and $y_{4}=s C_{4}$, the functions of inverse highpass filter and inverse differentiator can be realized at $V_{o 1}$ and $V_{o 2}$, respectively. They can be expressed by

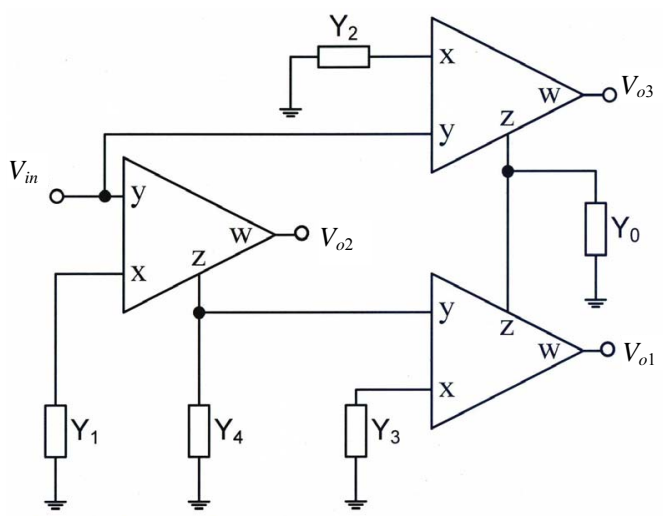

Figure 1. The proposed inverse filter scheme.

$$
\begin{gathered}
\frac{V_{o 1}}{V_{i n}}=\frac{V_{o 3}}{V_{i n}}=\frac{s^{2} C_{2} C_{4}+s C_{4} G_{2}+G_{1} G_{3}}{s^{2} C_{0} C_{4}} \\
\frac{V_{o 2}}{V_{i n}}=\frac{G_{1}}{s C_{4}}
\end{gathered}
$$

The output of $V_{o 3}$ has the same function as $V_{o 1}$, it provides the additional output which makes the filter application more flexible.

From (2) and (3), after the restricting ourselves only to the using of six passive elements, we can derive all the filter functions as shown in Table 1. It can be found that the coefficients of all terms in the numerator and denominator of the transfer functions are adjustable by independent passive elements. Furthermore, for the presented scheme in Figure 1, it can be observed that all the employed passive elements are grounded. The use of grounded passive elements conduces to easier electronic tunability and integrated-circuit implementation [10]. A number of realizations of tunable grounded passive elements can be found in the literature [10-13]. The passive sensitivities of corner angular frequency are equal to 0.5 for the inverse filter realizations in Table 1, so they can be classified as insensitive. In addition, the proposed configuration in Figure 1 possesses the characteristics of input and output cascadability due to its high input impedance and low output impedance. So it is convenient to connecting other stages at both input and output terminals for signal processing. It must be noted that the proposed inverse lowpass and inverse bandpass filters in [6] are included in the filter realizations of Table 1. The presented scheme in Figure 1 provides more flexible functions and different realization with identical configuration.

\section{Simulation Results}

To verify the potentialities of the proposed scheme, circuit simulations of the presented multi-function inverse filters have been carried out. The commercial current feedback amplifiers AD844 macromodel with $\pm 12 \mathrm{~V}$ voltage supply is used to realize the CFOA in Figure 1 [12]. Using an AD844 IC to realize the CFOA, its equivalent model can be shown in Figure 2. It is important to understand that the low input impedance at $\mathrm{x}$ ter-

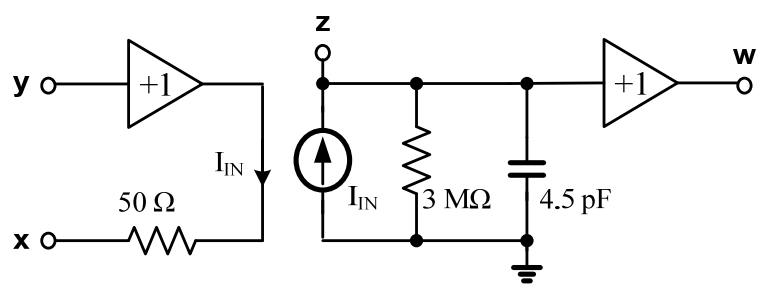

Figure 2. The realization of CFOA with an AD844 IC. 
Table 1. All the inverse filter functions using six passive elements.

\begin{tabular}{cccccccc}
\hline Case & Function at $V_{o 1}$ & Function at $V_{o 2}$ & $y_{0}$ & $y_{1}$ & $y_{2}$ & $y_{3}$ & $y_{4}$ \\
\hline 1 & Inverse lowpass & Differential & $G_{0}$ & $s C_{1}$ & $s C_{2}+G_{2}$ & $s C_{3}$ & $G_{4}$ \\
2 & Inverse lowpass & Inverse lowpass & $G_{0}$ & $s C_{1}+G_{1}$ & $G_{2}$ & $s C_{3}$ & $G_{4}$ \\
3 & Inverse lowpass & Differential & $G_{0}$ & $s C_{1}$ & $G_{2}$ & $s C_{3}+G_{3}$ & $G_{4}$ \\
4 & Inverse bandpass & Differential & $s C_{0}$ & $s C_{1}$ & $s C_{2}+G_{2}$ & $s C_{3}$ & $G_{4}$ \\
5 & Inverse bandpass & Inverse lowpass & $s C_{0}$ & $s C_{1}+G_{1}$ & $G_{2}$ & $s C_{3}$ & $G_{4}$ \\
6 & Inverse bandpass & Differential & $s C_{0}$ & $s C_{1}$ & $G_{2}$ & $s C_{3}+G_{3}$ & $G_{4}$ \\
7 & Inverse bandpass & Integration & $G_{0}$ & $G_{1}$ & $s C_{2}+G_{2}$ & $G_{3}$ & $s C_{4}$ \\
8 & Inverse bandpass & Integration & $G_{0}$ & $G_{1}$ & $s C_{2}$ & $s C_{3}+G_{3}$ & $s C_{4}$ \\
9 & Inverse bandpass & Inverse highpass & $G_{0}$ & $s C_{1}+G_{1}$ & $s C_{2}$ & $G_{3}$ & $s C_{4}$ \\
10 & Inverse highpass & Integration & $s C_{0}$ & $G_{1}$ & $s C_{2}$ & $s C_{3}+G_{3}$ & $s C_{4}$ \\
11 & Inverse highpass & Inverse highpass & $s C_{0}$ & $s C_{1}+G_{1}$ & $s C_{2}$ & $G_{3}$ & $s C_{4}$ \\
12 & Inverse highpass & Integration & $s C_{0}$ & $G_{1}$ & $s C_{2}+G_{2}$ & $G_{3}$ & $s C_{4}$ \\
\hline
\end{tabular}

minl is locally generated and does not depend on feedback. This is very different from the "virtual ground" of a conventional operational amplifier used in the current summing mode which is essentially an open circuit until the loop settles [8]. In the simulation, the values of all resistors and all capacitors are $40 \mathrm{k} \Omega$ and $1 \mathrm{nF}$, respectively.

It is found that the workability of all the inverse biquids in Table $\mathbf{1}$ is in good agreement with our theoretical prediction. The typical frequency responses of inverse lowpass (the case 1 of Table 1), inverse bandpass (the case 4 of Table 1) and inverse highpass (the case 12 of Table 1) are shown in Figure 3. The deviation to theoretical response is due to the parasitic impedance of nonideal CFOA [14].

\section{Conclusions}

We have proposed a novel scheme for the realization of an input and output cascadable voltage-mode multifunc-

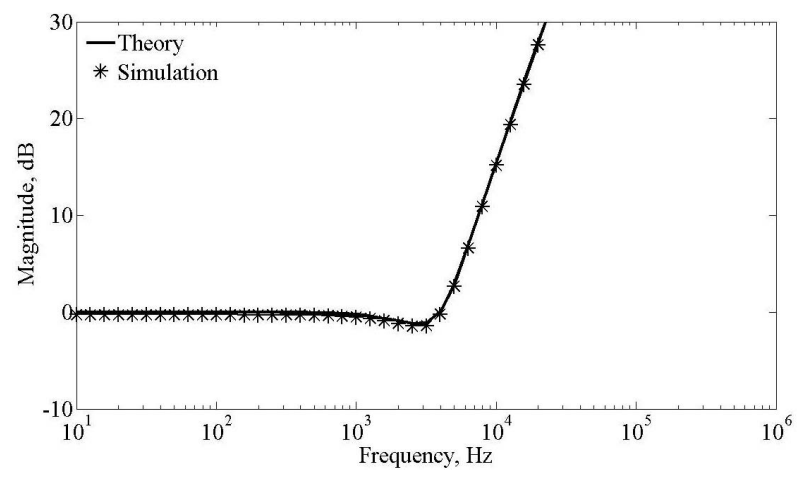

(a)

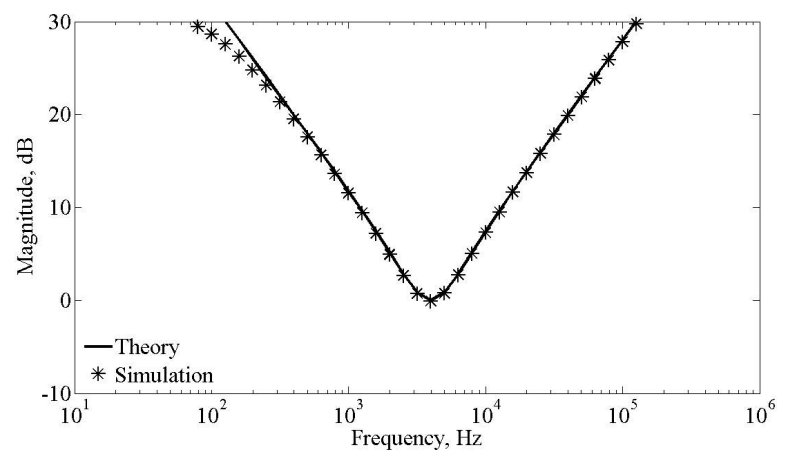

(b)

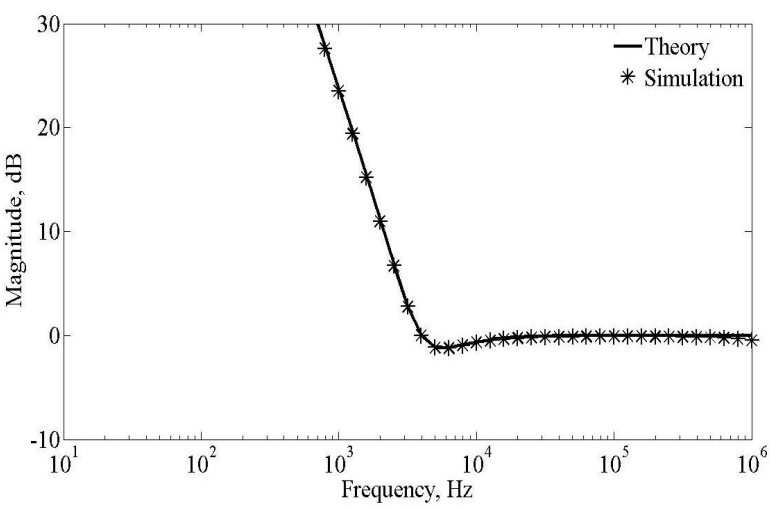

(c)

Figure 3. Typical frequency responses of inverse filters: (a) inverse lowpass; (b) inverse bandpass; (c) inverse highpass.

tion inverse filter. It consists of CFOAs and groundedpassive elements. Many various inverse filter functions are realized by slight modification of the passive elements of the proposed scheme. It offers more convenient 
realizations for inverse filter functions. The feasibility of the proposed circuit is verified by simulation results.

\section{References}

[1] A. Leuciuc, "Using Nullors for Realisation of Inverse Transfer Functions and Characteristics," Electronics Letters, Vol. 33, No. 11, 1997, pp. 949-951. doi:10.1049 /el: 19970637

[2] R. Kuc, "Introduction to Digital Signal Processing," McGraw-Hill, New York, 1988.

[3] B. Chipipop and W. Surakampontorn, "Realisation of Current-Mode FTFN-Based Inverse Filter," Electronics Letters, Vol. 35, No. 9, 1999, pp. 690-692. doi:10.10 49/el:19990495

[4] H. Y. Wang and C. T. Lee, "Using Nullors for Realisation of Current-Mode FTFN-Based Inverse Filters," Electronics Letters, Vol. 35, No. 22, 1999, pp. 1889-1890. doi:10.1049/el:19991336

[5] M. T. Abuelma'atti, "Identification of Cascadable Current-Mode Filters and Inverse-Filters Using Single FTFN," Frequenz, Vol. 54, No. 11, 2000, pp. 284-289.

[6] S. S. Gupta, D. R. Bhaskar, R. Senani and A. K. Singh, "Inverse Active Filters Employing CFOAs," Electrical Engineering, Vol. 91, No. 1, 2009, pp. 23-26. doi:10.10 07/s00202-009-0112-3

[7] G. H. Wang, Y. Fukui, K. Kubota and K. Watanabe, "Voltage-Mode to Current-Mode Conversion by an Extended Dual Transformation," IEEE Proceedings Inter- national Symposium on Circuits and Systems, Singapore, 11-14 June 1991, pp. 1833-1836.

[8] Analog Devices, $60 \mathrm{MHz} 2000 \mathrm{~V} / \mu \mathrm{s}$ Monolithic Op Amp AD844 Data sheet, Revision E, 2003. http://www. analog.com/static/imported-files/data_sheets/AD844.pdf

[9] A. Fabre, "Insensitive Voltage-Mode and Current-Mode Filters from Commercially Available Transimpedance Opamps," Circuits, Devices and Systems, IEE Proceedings G, Vol. 140, No. 5, 1993, pp. 319-321. doi:10.10 49/ip-g-2.1993.0053

[10] B. Nauta, "Analog CMOS Filters for Very High Frequencies," Kluwer Academic Publishers, Norwell, 1993.

[11] I. A. Khan and M. T. Ahmed, "OTA-Based Integrable Voltage/Current-Controlled Ideal C-Multiplier," Electronics Letters, Vol. 22, No. 7, 1986, pp. 365-366. doi:10. 1049/el:19860248

[12] K. Vavelidis and Y. Tsividis, "Design Considerations for a Highly Linear Electronically Tunable Resistor," ISCA '93, 1993 IEEE International Symposium on Circuits and Systems, Vol. 2, Chicago, 3-6 May 1993, pp. 1180-1183.

[13] A. Worapishet and P. Khumsat, "Sub-Threshold RMOSFET Tunable Resistor Technique," Electronics Letters, Vol. 43, No. 7, 2007, pp. 390-392. doi:10.1049/el: 20070175

[14] J. A. Svoboda, L. McGory and S. Webb, "Applications of a Commercially Available Current Conveyor," International Journal of Electronics, Vol. 70, No. 1, 1991, pp. 159-164. doi:10.1080/00207219108921266 